\title{
Kinematic Modelling of Multi-Terrain Pine Needle Collecting Robot (PiNCoR) for the Hilly areas of Uttarakhand
}

\author{
Shival Dubey*, Manish Prateek*, Mukesh Saxena** \\ *University of Petroleum and Energy Studies, Dehradun, India \\ **University of Technology and Management, Shillong, India
}

\begin{tabular}{l} 
Article Info \\
\hline Article history: \\
Received Apr 12, 2015 \\
Revised Jul 26, 2015 \\
Accepted Aug 10, 2015 \\
\hline Keyword: \\
Kinematic Modeling \\
Multi Terrain \\
Rocker Bogie Suspension \\
Mechanism \\
Wheeled Mobile Robot
\end{tabular}

\begin{abstract}
Uttarakhand - a northern state of India in the foothills of Himalayas has approximately $64.8 \%$ of forest. Pine (Pinus roxburghii) is an important tree species which is because of its numerous uses becomes more prominent in this hilly state. Pine Needle Collecting Robot (PiNCoR) is a machine designed to collect pine needles from the high altitude pine forest regions. The robot is capable to maneuver over rough terrains, can navigate through craters and can stock the collected pine needles. After analyzing the design constraints for navigation on the hilly terrains, a small rover was designed and the kinematics portion is discussed in this paper. With the use of a versatile rocker bogie suspension mechanism, this paper also presents the kinematic modelling of a wheeled mobile robot being the best for hilly areas with super stability and traction control being its assets. It analyzes the different inputs from the information provided by the on-board sensor system for maneuvering. The robot has five degrees of freedom $(\mathrm{DoF})$ providing it the flexibility to work in $\mathrm{x}$ and $\mathrm{z}$ axes as along with the standard pitch, roll and yaw directions.
\end{abstract}

Copyright @ 2015 Institute of Advanced Engineering and Science. All rights reserved.

\section{Corresponding Author:}

Shival Dubey,

University of Petroleum and Energy Studies,

Dehradun, India.

Email: shivaldubey@gmail.com

\section{INTRODUCTION}

Uttarakhand has four major forest types such as Oak, Deodar, Sal and Pine. There are majorly three community of Chirr Pine found here such as Sal-Pine, Pine pure stand and Oak Pine communities. Pine resin is one of the most important non-wood product from the pine apart from this each part of this plant such as Cone, Trunk, Stem, Wood, Leaves and bark are used by the indigenous community of the state [1] .Wheeled mobile robot has proved its utility over recent years in various areas such as military (bomb diffusion and rescue operations)[2-5], indoor and outdoor applications [6], industrial applications [7], medical services [8, 9] and planetary \& space exploration [10, 11]. The major advantage which makes these robots useful in various applications is their high mobility in different terrains and environments, which is a primary factor in performance evaluation of these machines.

The utility of a wheeled mobile robot has been proved in the recent past in space exploration by several scientific organizations including NASA, ISRO. There are several other applications of multi-terrain robots such as material handling, rescue operations and so on. The PinCoR needs to traverse in uneven terrain and therefore it has a relatively complex locomotion system. This is important to smoothly maneuver it to a longer distance along the uneven surface and through obstructions in the woods. In order to evaluate robot's actual location, orientation and its path trajectory; its kinematic modelling is significant. Due to its high mobility, it is quite challenging to determine the kinematic model, though a reasonably accurate forward kinematic analysis would be a fitting substitution. As the PiNCoR has an electrical energy source in the form of dry batteries, an efficient actuation plays an important part in its distant reach. 
Robot kinematics applies geometry to the study of the movement of multi-degree of freedom in the kinematic chains that form the structure of robotic systems. Although several researchers have developed different techniques for calculating kinematics of the robotic manipulators such as Jolly Shah [12] has shown kinematic analysis using neural networks, M. Gouasmi[13] has done Dual Quaternions method and so on. Forward kinematics specify the joint parameters and compute the configuration of the chain. For serial manipulators, this is accomplished by direct substitution of the joint parameters into the forward kinematic equations for the serial string. The forward kinematics determine the position and orientation, achieved by using Jacobian Matrices for each wheel of the robot. Since there is no steering, in order to nullify the effect of rotational slip on the tunings, inverse kinematics model was derived for the wheel rotational velocity.

\section{THE PINE NEEDLE COLLECTOR ROBOT - AN OUTLINE}

This section describes the robot characteristics for its kinematic modelling. The robot has six wheels using a rocker bogie suspension mechanism as shown in the Figure 1. The PiNCoR is $56.5 \mathrm{cms}$ long, 31.5 cms wide and $25.5 \mathrm{cms}$ high. The PiNCoR is equipped with a suction mechanism which enables the collection of pine needles from the ground. An adjustable ground clearance of $20 \mathrm{~cm}$ is provided which enables it to maneuver freely through pits and holes on the hills. All the wheels in PinCoR are $17 \mathrm{~cm}$ in diameter and have a nominal speed of $0.2 \mathrm{~m} / \mathrm{sec}$.

The smooth operation of the PiNCoR is due to the independently actuated wheels which are attached to a rocker bogie suspension mechanism which enables its movement in low velocity and uneven terrain. Rocker bogie suspension consists of two main rockers which are joined at a central differential unit, with the main body called as Averaging Mechanism. This stabilizes the body unit and helps in preventing the toppling effect of the rover at inclinations. Each rocker has three non-steerable independently actuated wheels; one at the front and the remaining two at the small rocker end in the back portion. Joint angle between the small and main rocker is denoted by $\psi_{1} \& \psi_{2}$, the left and right bogie angles (main rockers via differential) are denoted by $\beta_{1} \& \beta_{2}$. The wheel rotation is denoted by $\zeta_{\mathrm{i}}$ where $\mathrm{i}=1-6$. There are in total six actuators in the form of encoded motors connected to the wheels which enable independent movement of the PinCoR along the $\beta_{1}, \beta_{2}, \psi_{1}, \psi_{2}$ rocker bogie joints for flexibility based on the terrain.

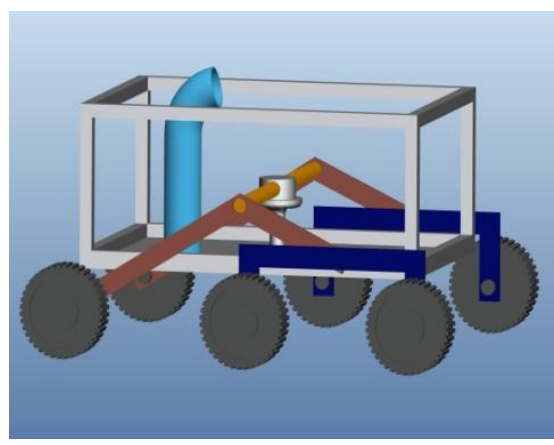

Figure 1. Pro - E Model of PinCoR

The encoded motors help in determining the wheel angular rotation $\zeta_{\mathrm{i}}$. The body roll, pitch and yaw was calculated with the help of accelerometer. The location of the rover in the forest was tracked using a real time GPS map at the base station and the PiNCoR equipped with battery level encoder so that it can return to base when the power reduces during its operation. In the coming sections, the forward kinematics of the PiNCoR using wheel Jacobian matrices has been discussed followed by the inverse kinematics derivation.

\section{FORWARD KINEMATICS}

For the forward kinematic analysis of the PiNCoR, the coordinate frame system for both left and right bogies was defined as shown in the Figure (2) \& (3). For the PiNCoR, a local coordinate system was defined and the origin of these coordinate system was called as 'Reference point' on the robot frame designated as ' $O$ ', the differential joint as ' $D$ ', the left and right bogie's as ' $\beta_{i}(i=1,2)$ ' and axes of all wheels as ' $A_{i}(i=1,2,3,4,5,6)$ '. Various design angles are shown in the Figure (2) \& (3). 


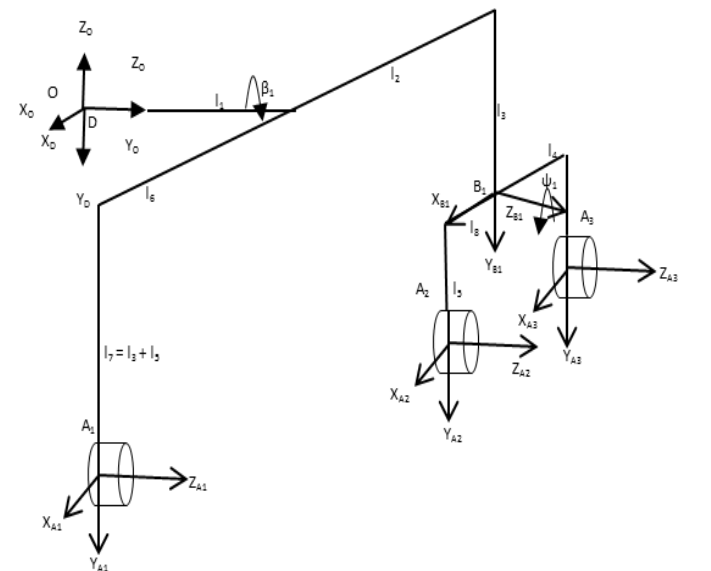

Figure 2. Left Coordinate Frame

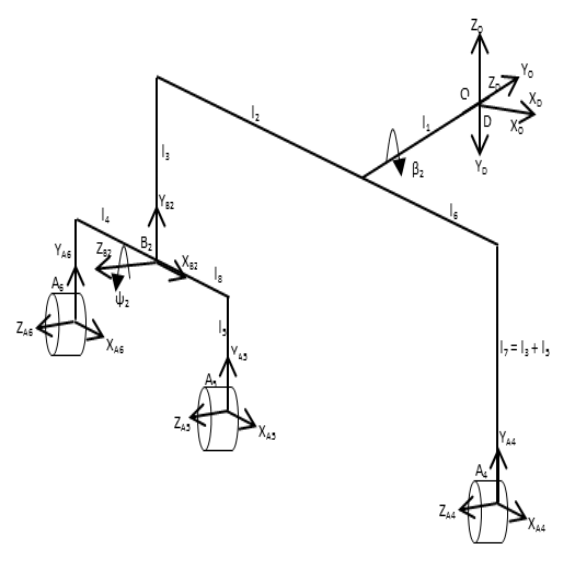

Figure 3. Right Coordinate Frame

Every coordinate frame shows a step by step kinematic chain from the origin to the wheel axis e.g. the kinematic transformation from the origin (reference) to the wheel 1 axle can be represented by $O-D-$ $A_{l}$. The coordinate frames was determined by the Denavit - Hertenberg notations [14] related to the various coordinates given in the Table 1 . The DH parameters define the transformations from one joint to the next joint in the kinematic chain, ' $\boldsymbol{\theta}$ ' defines rotation between the z-axis, ' $\boldsymbol{d}$ ' defines the distance along the z-axis between joints, ' $\boldsymbol{a}$ ' defines the distance along the x-axis between joints, and ' $\boldsymbol{\alpha}$ ' defines the angle between $\mathrm{z}$ axes of joints. The general transformation matrix is represented below based on the coordinate frame $i$ to coordinate frame $j$ using a homogenous transformation matrix:

$$
T_{j, i}=\left[\begin{array}{cccc}
\operatorname{Cos} \vartheta_{j} & -\operatorname{Sin} \vartheta_{j} \operatorname{Cos} \alpha_{j} & \operatorname{Sin} \vartheta_{j} \operatorname{Sin} \alpha_{j} & a_{j} \operatorname{Cos} \vartheta_{j} \\
\operatorname{Sin} \vartheta_{j} & \operatorname{Cos} \vartheta_{j} \operatorname{Cos} \alpha_{j} & -\operatorname{Cos} \vartheta_{j} \operatorname{Sin} \alpha_{j} & a_{j} \operatorname{Sin} \vartheta_{j} \\
0 & \operatorname{Sin} \alpha_{j} & \operatorname{Cos} \alpha_{j} & d_{j} \\
0 & 0 & 0 & 1
\end{array}\right]
$$

Where $\vartheta_{j}, \alpha_{j}, a_{j}, d_{j}$ are the $D-H$ parameters for coordinate frame $\mathrm{j}$. The above matrix shows the transformation of coordinate frame $\mathrm{j}$ in terms of coordinate frame $\mathrm{i}$. The transformations from the robot reference frame ' $O$ ' to the wheel axle ' $A_{l}$ ' was obtained by cascading each transformation.

$$
\mathbf{T}_{\mathbf{O}, \mathrm{A} 1}=\mathbf{T}_{\mathbf{O}, \mathrm{D}} \mathbf{T}_{\mathrm{D}, \mathrm{B} 1} \mathbf{T}_{\mathrm{B} 1, \mathrm{~A} 1}
$$

Table 1: D - H Parameters

\begin{tabular}{ccccc}
\hline Joint Axis & $\vartheta(\mathrm{deg})$ & $\alpha(\mathrm{deg})$ & $\mathrm{d}(\mathrm{cm})$ & $\mathrm{a}(\mathrm{cm})$ \\
\hline $\mathrm{O}-\mathrm{D}$ & 0 & -90 & 0 & 0 \\
$\mathrm{D}-\mathrm{A}_{1}$ & $\beta_{1}$ & 0 & 15 & 44 \\
$\mathrm{D}-\mathrm{B}_{1}$ & $\beta_{1}$ & 0 & 15 & -19 \\
$\mathrm{~B}_{1}-\mathrm{A}_{2}$ & $\Psi_{1}$ & 0 & 0 & 20 \\
$\mathrm{~B}_{1}-\mathrm{A}_{3}$ & $\Psi_{1}$ & 0 & 0 & -20 \\
$\mathrm{D}-\mathrm{A}_{2}$ & $\beta_{2}$ & 180 & -15 & 44 \\
$\mathrm{D}-\mathrm{B}_{2}$ & $\beta_{2}$ & 90 & -15 & -19 \\
$\mathrm{~B}_{2}-\mathrm{A}_{2}$ & $\Psi_{2}$ & 0 & 0 & 20 \\
$\mathrm{~B}_{2}-\mathrm{A}_{3}$ & $\Psi_{2}$ & 0 & 0 & -20 \\
\hline
\end{tabular}

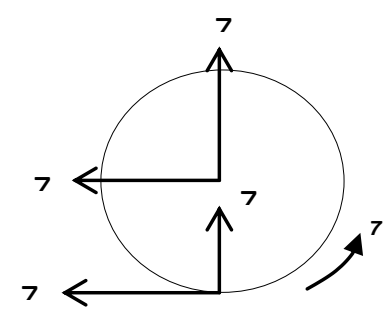

Figure 4. Wheel Motion Frame 
To capture the wheel motion, there is an additional requirement of coordinate frames i.e. wheel contact frame $C_{i}$ and wheel motion frame $M_{i}$. To obtain $C_{i}$, the wheel axis $A_{i}$ was rotated about the $Z$ - axis until the $\mathrm{X}$ - axis became parallel to the ground which was later rotated by an angle of 90 degrees.

The derivation of the transformation matrices of contact frame $\mathrm{C}_{\mathrm{i}}$ was done using Euler angle Z-X-Y as given below.

$\mathbf{T}_{\mathbf{A} \mathbf{i}, \mathbf{C i}}=\left[\begin{array}{cccc}\operatorname{Cos} p_{i} \operatorname{Cos} r_{i}-\operatorname{Sin} p_{i} \operatorname{Sinq}_{i} \operatorname{Sinr}_{i} & \operatorname{Cos} r_{i} \operatorname{Sin} p_{i}+\operatorname{Cos} p_{i} \operatorname{Sin} q_{i} \operatorname{Sin} r_{i} & -\operatorname{Cos} q_{i} \operatorname{Sin} r_{i} & 0 \\ -\operatorname{Cos} q_{i} \operatorname{Sin} p_{i} & \operatorname{Cos} p_{i} \operatorname{Cos} q_{i} & \operatorname{Sin} q_{i} & 0 \\ \operatorname{Cos} r_{i} \operatorname{Sin} p_{i} \operatorname{Sin} q_{i}+\operatorname{Cos} p_{i} \operatorname{Cos} r_{i} & -\operatorname{Cos} p_{i} \operatorname{Cos} r_{i} \operatorname{Sin} q_{i}+\operatorname{Sin} p_{i} \operatorname{Sin} r_{i} & \operatorname{Cos} q_{i} \operatorname{Cos} r_{i} & 0 \\ 0 & 0 & 0 & 1\end{array}\right]$

Where $\mathrm{p}_{\mathrm{i}}, \mathrm{q}_{\mathrm{i}}, \& \mathrm{r}_{\mathrm{i}}$ are the rotation angle about $\mathrm{X}, \mathrm{Y}$ and $\mathrm{Z}$ respectively.

Also, in order to obtain the wheel motion frame, it was translated along the negative $\mathrm{Z}-$ axis by wheel radius $\left(\mathrm{R}_{\mathrm{w}}\right)$ and translating along the $\mathrm{X}-$ axis for wheel roll $\left(\mathrm{R}_{\mathrm{w}} \zeta_{\mathrm{i}}\right)$.

Hence the transformation matrices, for all frames, can be shown as below:

$$
\begin{aligned}
& T_{O M 1}=T_{O D}, T_{D A 1}, T_{A 1 C 1}, T_{C 1 M 1} \\
& T_{O M 2}=T_{O D}, T_{D B 1}, T_{B 1 A 2}, T_{A 2 C 2}, T_{C 2 M 2} \\
& T_{O M 3}=T_{O D}, T_{D B 1}, T_{B 1 A 3}, T_{A 3 C 3}, T_{C 3 M 3} \\
& T_{O M 3}=T_{O D}, T_{D A 4}, T_{A 4 C 4}, T_{C 4 M 4} \\
& T_{O M 4}=T_{O D}, T_{D B 2}, T_{B 2 A 5}, T_{A 5 C 5}, T_{C 5 M 5} \\
& T_{O M 6}=T_{O D}, T_{D B 2}, T_{B 2 A 6}, T_{A 6 C 6}, T_{C 6 M 6}
\end{aligned}
$$

To obtain the wheel Jacobian matrix, the motion of the robot must be expressed as wheel motion by applying the derivative of instantaneous transformation from the origin ' $\boldsymbol{O}$ ' to the wheel motion ' $\boldsymbol{M}_{\boldsymbol{i}}$ ' $\dot{\mathrm{T}}_{\widehat{O}, \widehat{M}_{l}}$ as shown below

$$
\dot{T}_{\widehat{O}, R}=\dot{T}_{\widehat{O}, \bar{M}_{l}} \dot{T}_{\left(M_{i}\right),(O)} \quad i=1,2,3,4,5,6
$$

where the derivative of the transformation matrices and instantaneous matrices was discussed in [15]. Using Euler angles, Yaw $\varphi$ (rotation about $\mathrm{Z}$ - axis), Roll $\mathrm{r}$ (rotation about $\mathrm{X}-$ axis) and Pitch $\mathrm{p}$ (rotation about $\mathrm{Y}-$ axis) can be derived. From the eq. (9), the derivative of the robot coordinate frame $\dot{T}_{\widehat{O}, \widehat{M}_{l}}$ was as following

$$
\dot{T}_{\widehat{O}, \widehat{M}_{l}}=\left[\begin{array}{cccc}
0 & -\dot{\varphi} & \dot{p} & \dot{x} \\
\dot{\varphi} & 0 & \dot{-r} & \dot{y} \\
\dot{-p} & \dot{r} & 0 & \dot{z} \\
0 & 0 & 0 & 1
\end{array}\right]
$$

The transformations in eq. (10) can be derived by solving equations $(3-9)$ for each wheel in addition to the table 1 consisting of the D - H Parameters. Once the derivative is obtained for each wheel, it gives an equation set for robot's motion to the joint angular rates in vector form $\left[\begin{array}{llllll}\dot{x} & \dot{y} & \dot{z} & \dot{\varphi} & \dot{p} & \dot{r}\end{array}\right]^{\mathrm{T}}$. The resultants for wheel 1 and 4 (left \& right front) were

$$
\left[\begin{array}{c}
\dot{x} \\
\dot{y} \\
\dot{z} \\
\dot{\varphi} \\
\dot{p} \\
\dot{r}
\end{array}\right]=\left[\begin{array}{ccc}
R_{w} \operatorname{Cos} \beta & 0 & b_{i} d_{s 1} \cos \beta \\
R_{w} & 0 & a_{s 1} \\
b_{i} R_{w} \sin \beta & 0 & d_{1} \operatorname{Sin} \beta \\
0 & 0 & -\cos \beta \\
0 & b_{i} & 0 \\
0 & 0 & -b_{i} \sin \beta
\end{array}\right]\left[\begin{array}{c}
\dot{\zeta_{l}} \\
\dot{\beta} \\
\eta_{i}
\end{array}\right] \text { for } i=1,4
$$

Where $b_{1}=-1$ and $b_{2}=1, a_{s 1}$ and $d_{s 1}$ are kinematic parameters defined in $\mathrm{D}-\mathrm{H}$ parameters of Table 1 , and $\mathrm{R}_{\mathrm{w}}$ is Wheel Radius. Though the wheels are non - steerable, the rotational slip must be considered. Hence $\eta_{\mathrm{i}}$ is the wheel rotational slip for (1-6) wheels. The middle wheels 2 and 3 have same kinematic equations as shown below 
$\left[\begin{array}{c}\dot{x} \\ \dot{y} \\ \dot{z} \\ \dot{\varphi} \\ \dot{p} \\ \dot{r}\end{array}\right]=\left[\begin{array}{cccc}R_{w} \operatorname{Cos} \llbracket\left(\sigma \rrbracket_{1}\right) & 0 & -a_{\gamma 1} \sin \llbracket\left(\vartheta \rrbracket_{\gamma 1}\right) & -d_{s 1} \cos \llbracket\left(\sigma \rrbracket_{1}\right) \\ 0 & 0 & 0 & K_{i} \\ -R_{w} \sin \left(\sigma_{1}\right) & 0 & -a_{\gamma 1} \cos \left(\vartheta_{\gamma 1}\right) & d_{s 1} \sin \left(\sigma_{1}\right) \\ 0 & 0 & 0 & -\cos \left(\sigma_{1}\right) \\ 0 & -1 & -1 & 0 \\ 0 & 0 & 0 & -\sin \left(\sigma_{1}\right)\end{array}\right]\left[\begin{array}{c}\dot{\zeta}_{l} \\ \dot{\beta} \\ \dot{\gamma_{1}} \\ \eta_{i}\end{array}\right]$ for $i=2,3$

Where $\sigma_{1}=\gamma_{1}+\beta, \gamma_{1}$ is the left rocker angle, $\mathrm{K}_{\mathrm{i}}=\mathrm{a}_{\mathrm{Ai}} \cos \left(\vartheta_{\mathrm{ci}}\right)+\mathrm{a}_{\gamma 1} \cos \left(\vartheta_{\mathrm{ci}}+\vartheta_{\mathrm{Ai}}\right) ; \mathrm{i}=2,3, \mathrm{~d}_{\mathrm{s} 1}, \mathrm{a}_{\mathrm{A} 3}, \vartheta_{\mathrm{ci}}, \mathrm{a}_{\gamma \mathrm{i}}$ and $\gamma_{\mathrm{Ai}}$ are the kinematic parameter provided in the table 1

The back wheels 5 and 6 have similar kinematic equations as equation number (12):

$$
\left[\begin{array}{c}
\dot{x} \\
\dot{y} \\
\dot{z} \\
\dot{\varphi} \\
\dot{p} \\
\dot{r}
\end{array}\right]=\left[\begin{array}{cccc}
R_{w} \cos \llbracket\left(\sigma \rrbracket_{2}\right) & 0 & -a_{\gamma 1} \sin \llbracket\left(\vartheta \rrbracket_{\gamma 2}\right) & \left.-d_{s 1} \cos \llbracket(\sigma)_{2}\right) \\
0 & 0 & 0 & K_{i} \\
-R_{w} \sin \left(\sigma_{2}\right) & 0 & -a_{\gamma 1} \cos \left(\vartheta_{\gamma_{2}}\right) & d_{s 1} \sin \left(\sigma_{2}\right) \\
0 & 0 & 0 & -\cos \left(\sigma_{2}\right) \\
0 & -1 & -1 & 0 \\
0 & 0 & 0 & -\sin \left(\sigma_{1}\right)
\end{array}\right]\left[\begin{array}{c}
\dot{\zeta}_{l} \\
\dot{\beta} \\
\dot{\gamma_{2}} \\
\eta_{i}
\end{array}\right] \text { for } i=5,6
$$

Where $\sigma_{2}=\gamma_{2}-\beta, \gamma_{2}$ is the right angle angle, $K_{i}=a_{A i} \cos \left(\vartheta_{c i}\right)+a_{y 2} \cos \left(\vartheta_{c i}+\vartheta_{A i}\right) ; i=5$,

The general form of the set of equations from $(11-13)$ is $\dot{u}=J_{i} \dot{q}_{\imath} \quad \mathrm{i}=1-6$; where $\mathrm{J}$ is the Jacobian matrix of the wheel $i$, and $\dot{q}$ is the Joint angular rate vector.

\section{INVERSE KINEMATICS}

Inverse kinematic model is required to determine the velocity of individual wheel in order to accomplish desired rover motion. The motion of the robot will be given by the forward velocity. Since there is no steering, there will be no turning rate or rotational slip. The actuation velocity of all the wheels of PinCoR was developed. Since there is no steering in any of the wheel, the wheel rolling velocity of the robot has been discussed below.

\subsection{Wheel Rolling Velocities}

Let us assume that the desired forward velocity and the angular rate of the rover are $\dot{x_{d}}$ and $\dot{\varphi}_{d}$ respectively. The equation set by solving eq. (11) would be:

$$
\begin{aligned}
& \dot{x_{d}}=\mathrm{R}_{\mathrm{w}} \cos (\beta) \dot{\zeta}_{l}+\mathrm{b}_{\mathrm{i}} \mathrm{d}_{\mathrm{s} 1} \cos (\beta) \dot{\eta}_{l} \\
& \dot{\varphi}_{d}=-\cos (\beta) \dot{\eta}_{l}
\end{aligned} \quad i=1,2
$$

Equation (14) would then give the wheel rolling velocity of wheel 1 and 2 by solving the two equations:

$$
\dot{\zeta}_{1}=\frac{\dot{x_{d}}-\dot{d}_{s 1} \dot{\varphi_{d}}}{R_{w} \operatorname{Cos} \beta}
$$

In the same way, the rolling velocities of wheel 3 and 5 was derived using eq. (12)

$$
\begin{aligned}
& \dot{x_{d}}=\mathrm{R}_{\mathrm{w}} \cos (\beta) \dot{\zeta}_{l}-\mathrm{a}_{\gamma 1} \sin \left(\vartheta_{\gamma 1}\right) \gamma_{1}-\mathrm{d}_{\mathrm{s} 1} \cos \left(\sigma_{1}\right) \dot{\eta}_{l} \\
& \dot{\varphi}_{d}=-\cos \left(\sigma_{1}\right) \dot{\eta}_{l} \\
& i=3,5
\end{aligned}
$$

Equation (16) will provide the rolling velocities of wheel 3 and 5;

$$
\dot{\zeta}_{1}=\frac{\dot{x_{d}}-d_{s 1} \dot{\varphi_{d}}+\dot{a}_{\gamma 1} \sin \left(\vartheta_{\gamma 1}\right) \gamma_{1}}{R_{w} \operatorname{Cos} \sigma_{1}} \quad i=3,5
$$

Finally the rolling velocity of wheel 4 and 6 from equation number (13) can be written as: 


$$
\dot{\zeta}_{1}=\frac{\dot{x}_{d}+d_{s 1} \dot{\varphi_{d}}+\dot{a}_{\gamma 1} \sin \left(\vartheta_{\gamma 2}\right) \gamma_{2}}{R_{w} \operatorname{Cos} \sigma_{2}} \quad i=4,6
$$

These wheel rolling velocities equations are relatively simple and do not require any complex matrix operations as in forward kinematics. Further the solution of the eq. $(15,17$ and 18) can be defined by the operating range of kinematic parameters such as $\beta, \sigma_{1}$ and $\sigma_{2}$.

\section{CONCLUSIONS}

A high mobility Kinematic modeling of PiNCoR is discussed in this paper. The approach used is different from the conventionally used methods which are only applicable to the mobile robots working on flat and smooth surfaces. The paper also shares the Denavit - Hartenberg notation being the serial link manipulator for the rocker bogie suspension system for the locomotion of the robot. For the kinematic calculations, the method used is simple and modified according to the configuration of the Pine Needle Collector Robot (PiNCoR). The D - H Parameters were applied and the results obtained was used to optimize the kinematics and for efficient driving of the wheels.

\section{ACKNOWLEDGEMENT}

This work is carried out and supported by the Research and Development Department of University of Petroleum and Energy Studies, Dehradun. I would like to thank Mr. Venkateswaran PS - Research Scientist UPES, for his kind help in reviewing and amending this article. I also acknowledge the work done by Mr. Prashant Shukla - Assistant Professor, UPES in developing the software model of the prototype.

\section{REFERENCES}

[1] Kala, C.P., Indigenous uses and structure of chir pine forest in Uttaranchal Himalaya, India. International Journal of Sustainable Development \& World Ecology, 2004. 11(2): p. 205-210.

[2] Murphy, R.R., Human-robot interaction in rescue robotics. Systems, Man, and Cybernetics, Part C: Applications and Reviews, IEEE Transactions, 2004. 34(2): p. 138-153.

[3] Weiner, T., A new model army soldier rolls closer to the battlefield. New York Times, 2005. 16: p. A1.

[4] Sharkey, N., Grounds for discrimination: Autonomous robot weapons. RUSI Defence Systems, 2008. 11(2): p. 86-89.

[5] Pastore, T.H., H. Everett, and K. Bonner, Mobile robots for outdoor security applications, 1999, DTIC Document.

[6] Noreils, F.R., Toward a robot architecture integrating cooperation between mobile robots: Application to indoor environment. International Journal of Robotics Research, 1993. 12(1): p. 79-98.

[7] Groover, M.P., M. Weiss, and R.N. Nagel, Industrial Robotics: Technology, Programming and Application 1986: McGraw-Hill Higher Education.

[8] Marohn, C.M.R. and C.E.J. Hanly, Twenty-first century surgery using twenty-first century technology: surgical robotics. Current Surgery, 2004. 61(5): p. 466-473.

[9] Ewing, D.R., et al., Robots in the operating room — the history. Surgical Innovation, 2004. 11(2): p. 6371.

[10] Bogue, R., Robots for space exploration. Industrial Robot. International Journal, 2012. 39(4): p. 323328.

[11] Elfes, A., et al. Safe and Efficient Robotic Space Exploration with Tele-Supervised Autonomous Robots. in AAAI Spring Symposium: To Boldly Go Where No Human-Robot Team Has Gone Before. 2006.

[12] Shah, J.A., S. Rattan, and B.C. Nakra, Kinematic Analysis of 3-DOF Planer Robot Using Artificial Neural Network. IAES International Journal of Robotics and Automation (IJRA), 2012. 1(3): p. 145 151.

[13] Gouasmi, M., Robot Kinematics, using Dual Quaternions. IAES International Journal of Robotics and Automation (IJRA), 2012. 1(1): p. 13-30.

[14] Craig, J.J., Introduction to robotics: mechanics and control. Vol. 3. 2005: Pearson Prentice Hall Upper Saddle River.

[15] Muir, P.F. and C.P. Neuman, Kinematic modeling of wheeled mobile robots. Journal of robotic systems, 1987. 4(2): p. 281-340. 